\title{
The M6 risk prediction model and two-step strategy to characterize pregnancies of unknown location: a multicentre external validation study
}

Evangelia Christodoulou ${ }^{1}$, Shabnam Bobdiwala ${ }^{2}$, Christopher Kyriacou ${ }^{2}$, Jessica Farren ${ }^{3}$, Nicola Mitchell-Jones ${ }^{4}$, Francis Ayim ${ }^{5}$, Baljinder Chohan ${ }^{6}$, Osama Abughazza ${ }^{7}$, Bramara Guruwadahyarhalli $^{8}$, Maya Al-Memar ${ }^{2}$, Sharmista Guha ${ }^{8}$, Veluppillai Vathanan ${ }^{6}$, Debbie Gould $^{3}$, Catriona Stalder ${ }^{2}$, Laure Wynants ${ }^{1,9}$, Dirk Timmerman ${ }^{1}$, Tom Bourne ${ }^{1,10}$, and Ben Van Calster ${ }^{1,11}$

${ }^{1} \mathrm{KU}$ Leuven

${ }^{2}$ Queen Charlotte's and Chelsea Hospital

${ }^{3}$ St Mary's Hospital

${ }^{4}$ Chelsea and Westminster NHS Trust

${ }^{5}$ Hillingdon Hospital

${ }^{6}$ Wexham Park Hospital

${ }^{7}$ Royal Surrey County Hospital NHS Foundation Trust

${ }^{8}$ Chelsea and Westminster Healthcare NHS Trust

${ }^{9}$ Maastricht University

${ }^{10}$ Imperial College London

${ }^{11}$ Leiden University Medical Centre

April 28, 2020

\begin{abstract}
Objective. To externally validate the M6 risk model and the two-step triage strategy (2ST) to triage pregnancies of unknown location (PUL), and compare performance with the M4 model and beta human chorionic gonadotropin (BhCG) ratio cut-offs. Design. Model validation study. Setting. Eight UK hospitals with early pregnancy assessment units. Population. Women presenting with a PUL and BhCG $>25 \mathrm{IU} / \mathrm{L}$. Methods. Women were managed using the 2ST protocol: step 1 classifies PUL as low risk of ectopic pregnancy (EP) if presenting progesterone [?]2 nmol/L, M6 is used as step 2 in the remaining cases. We validated 2ST and M6 alone (with and without progesterone as a predictor: M6P and M6NP). M6 and M4 require the BhCG ratio over two days. Based on these models, we classified PUL as high risk for EP when the risk was [?]5\%. We meta-analysed centre-specific results. Main outcome measures. Discrimination, calibration and clinical utility (decision curve analysis) for predicting EP. Results. Of 2899 eligible women, the main analysis excluded 297 (10\%) women that were lost to follow-up. $16 \%$ (95\% confidence interval 12-20) of women had presenting progesterone [?]2 nmol/L. The area under the ROC curve for EP was 0.88 (0.86-0.90) for 2ST and 0.89 (0.86-0.91) for M6P. Sensitivity for EP was $94 \%$ (89\%-97\%) for 2ST and 96\% (91\%-98\%) for M6P. Both approaches had good overall calibration, with modest variability between centres. M4 and BhCG ratio cut-offs had inferior performance and lower clinical utility. Conclusions. The 2ST and M6P alone are the best approaches to triage PUL.
\end{abstract}

\section{Hosted file}

MAIN DOCUMENT_M6 external validation.06.04.20.doc available at https://authorea.com/users/309444/ 
articles/440378-the-m6-risk-prediction-model-and-two-step-strategy-to-characterize-pregnanciesof-unknown-location-a-multicentre-external-validation-study

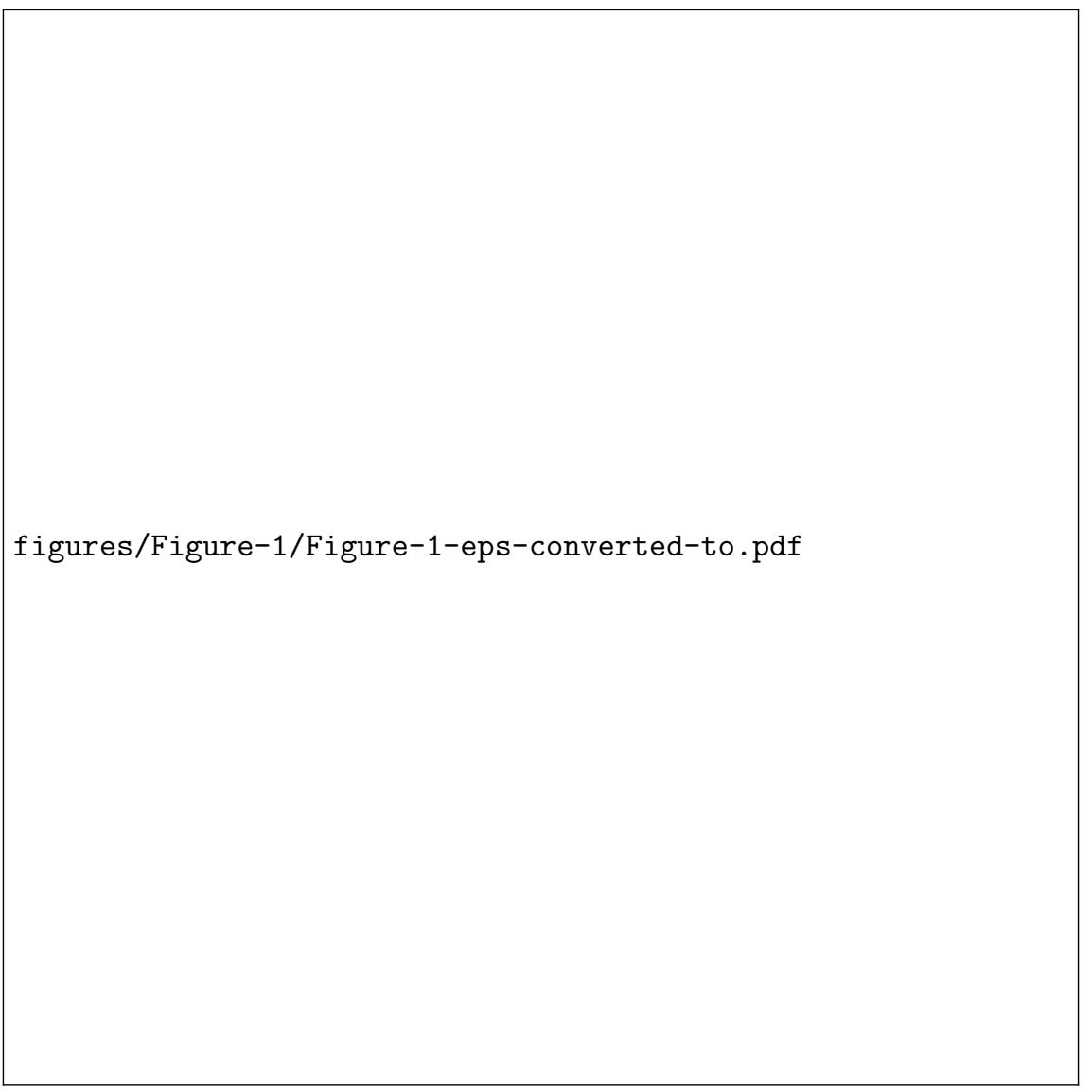


figures/Figure-2/Figure-2-eps-converted-to.pdf 